\title{
GO Synthesis of Offset Dual Reflector Antennas Using Local Axis-Displaced Confocal Quadrics
}

\author{
Aline R. Assis ${ }^{1,4} \odot$, Fernando J. S. Moreira ${ }^{2} \bullet$, José R. Bergmann ${ }^{3} \bullet$ \\ ${ }^{1}$ Graduate Program in Electrical Engineering, Federal University of Minas Gerais, Av. Pres. Antonio \\ Carlos 6627, 31270-901, Belo Horizonte, MG, Brazil \\ ${ }^{2}$ Department of Electronics Engineering, Federal University of Minas Gerais, Av. Pres. Antonio \\ Carlos 6627, 31270-901, Belo Horizonte, MG, Brazil \\ ${ }^{3}$ Centro de Estudos em Telecomunicações, Pontifícia Universidade Católica do Rio de Janeiro \\ (CETUC PUC-Rio), Rua Marquês de São Vicente 225, 22451-900, Rio de Janeiro RJ, Brazil, \\ ${ }^{4}$ Departamento de Engenharia Elétrica, Universidade Federal de Ouro Preto, Rua Trinta e Seis 115, \\ 35931-008, João Monlevade, MG, Brazil \\ aline@ufop.edu.br,fernandomoreira@ufmg.br,bergmann@puc-rio.br
}

\begin{abstract}
This work investigates an alternative numerical scheme for the solution of an exact formulation based on Geometrical Optics (GO) principles to synthesize offset dual reflector antennas. The technique is suited to solve a second-order nonlinear partial differential equation of the Monge-Ampère type as a boundary value problem. An iterative algorithm based on Newton's method was developed, using axis-displaced confocal quadrics to locally represent the subreflector surface, thus enabling an analytical description of the partial derivatives within the formulation. Such approach reduces discretization errors, as exact expressions for the mapping function and its derivatives are analytically determined. To check the robustness of the methodology, an offset dualreflector Gregorian antenna was shaped to provide a Gaussian aperture field distribution with uniform phase within a superelliptical contour. The shaped surfaces were further interpolated by quintic pseudo-splines and analyzed by Physical Optics (PO) with equivalent edge currents to validate the synthesis procedure at $11,725 \mathrm{GHz}$.
\end{abstract}

Index Terms - Offset dual-reflector antennas, geometrical optics (GO), Monge-Ampère equation, axis-displaced confocal quadrics.

\section{INTRODUCTION}

High efficiency, low sidelobes levels, and low cross-polarization are requirements desired in a wide variety of communication systems, as in satellite communications [1], radio astronomy, and radar [2]. An efficient way to achieve high performance is by shaping reflector antennas. In the 1970s and 1980s, several papers presented exact formulations based on Geometrical Optics (GO) to shape offset single and dual reflectors [2]-[4]. The formalism led to second-order nonlinear partial differential equations of the Monge-Ampère type, which were derived by simultaneously imposing Snell's Law and conservation of energy in the tube of reflected rays. Although the existence and uniqueness of the solution to the Monge-Ampère equation have not been mathematically proved, the equation can be numerically solved as a boundary value problem [2]-[4]. For instance, in [2] a finite-difference scheme associated with regular grids is employed to approximately deal with the derivatives. Then, an Brazilian Microwave and Optoelectronics Society-SBMO received 24 Nov 2019; for review 28 Nov 2019; accepted 3 Mar 2020 Brazilian Society of Electromagnetism-SBMag 
iterative algorithm solves a linearized form of the Monge-Ampère equation [2], [5], [6].

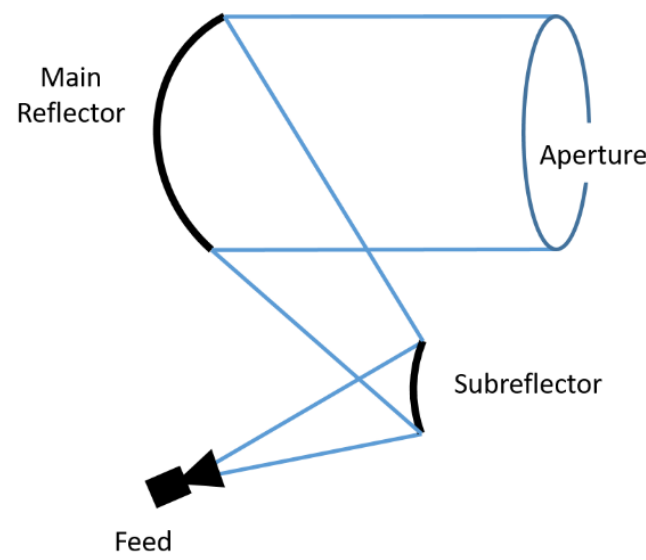

(a)

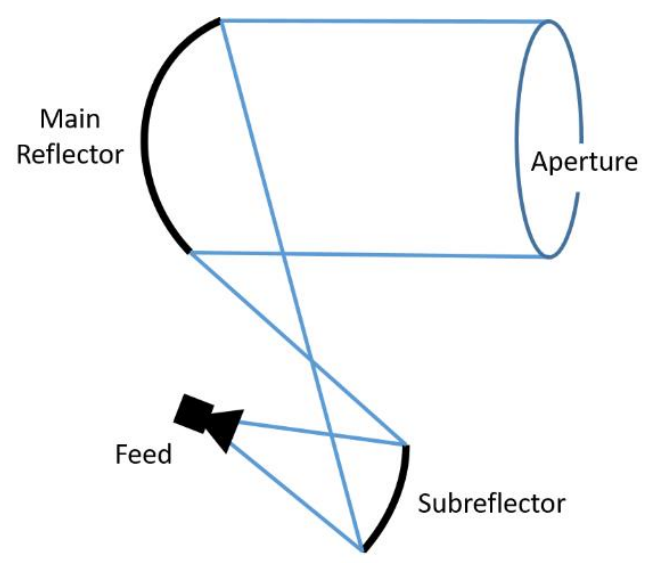

(b)

Fig. 1. Offset dual-reflector antennas: (a) Cassegrain and (b) Gregorian configurations.

Although the shaping of offset reflectors is well established in the literature, recently Penchel et al. [7] presented a rigorous numerical solution for the shaping of a single offset reflector. Unlike [2], [5] and [6], which adopted iterative numerical procedures with finite differences to linearize the differential operator, [7] used axis displaced confocal quadrics to locally represent the offset reflector surface. Such approach reduced discretization errors associated to partial derivatives, since exact expressions of the mapping function and its derivatives were analytically determined in terms of the computed quadrics' parameters.

The present work explores and extends the methodology developed in [7] to shape offset dualreflector antennas (see Fig. 1). To reach its purpose, the work is organized as follows. In Section II the complex notation describing an optical ray direction is presented. In Section III the synthesis problem formulated in [2], [4] and its boundary conditions are discussed. Section IV presents the simplifications of the mapping function and the Monge Ampère equation, associated to the use of confocal quadrics to locally describe the subreflector's surface. In Section V the numerical scheme used to solve the Monge-Ampère equation is explained. Finally, a case study is investigated in Section VI to validate the proposed shaping method.

\section{VECTOR DIRECTION IN COMPLEX COORDINATES}

The dual-reflector shaping investigated in this work is based on GO principles. When dealing with geometric surfaces, it is more appropriate to use stereographic projections to represent the directions of incident and reflected rays. This formalism permits to transform trigonometric expressions into polynomials expressions in the synthesis formulation and, consequently, some relations become explicit [8]. A unit vector $\hat{p}=\overrightarrow{O P}$ can be parametrized by rectangular coordinates $(u, v)$ using the 
stereographic projection of point $P$ from $N(0,0,1)$ in a point $P^{\prime}$ on the plane $z=0$ (see Figure 2).

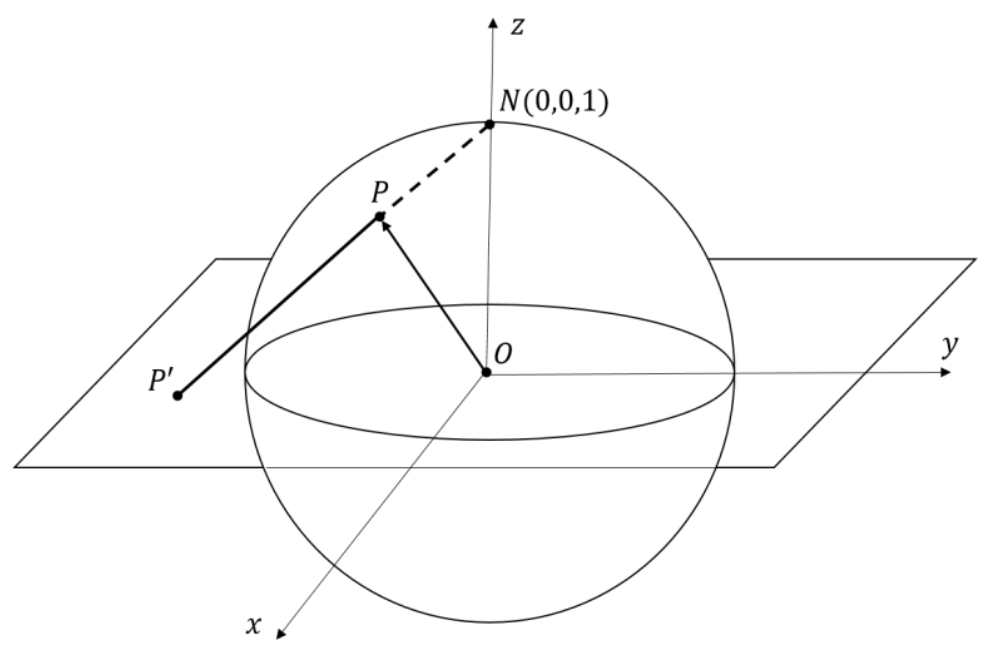

Fig. 2. Stereographic projection of a point $\mathrm{P}$.

The relation between the complex coordinate $\eta=u+i v$ associated with $P^{\prime}$ and the spherical coordinates $\theta$ and $\phi$ of point $P$ is given by [8]:

$$
\eta=\cot \left(\frac{\theta}{2}\right) e^{i \phi}
$$

From (1), is straightforward to show that:

$$
\begin{gathered}
\mathrm{e}^{i \phi} \sin \theta=\frac{2 \eta}{|\eta|^{2}+1} \\
\cos \theta=\frac{|\eta|^{2}-1}{|\eta|^{2}+1}
\end{gathered}
$$

where $|\eta|$ is the modulus of $\eta$. Consequently, a general vector $\vec{a}$ with Cartesian components $(a x, a y, a z)$ can be represented as $(\gamma, a z)$ where $\gamma$ is the complex number $\gamma=a x+i a y$. Using this representation, and from (2a) and (2b), the unit vector $\hat{p}=\overrightarrow{O P}$ can be written with respect to its complex coordinate $\eta$ as

$$
\hat{p}=\left(\frac{2 \eta}{|\eta|^{2}+1}, \frac{|\eta|^{2}-1}{|\eta|^{2}+1}\right)
$$

\section{GO SYNTHESIS: PROBLEM DESCRIPTION}

The basic geometry of the dual-reflector GO synthesis is illustrated in Fig. 3, where only the local quadric surfaces are shown. A ray emanating from the source (feed) phase center $O$ (which is also the origin of the coordinate system) is reflected at the subreflector point $R$ and then at the main-reflector point $S$, passing through the aperture plane $z=d$ at point $Q$ afterwards. The directions of the optical path segments $O R$ and $R S$ are represented by $\vec{r}(\eta)$ and $\vec{s}(\xi)$, respectively, and parametrized by complex coordinates $\eta$ and $\xi$, respectively. Furthermore, the coordinates of $Q(\omega, d)$ at the aperture plane are parametrized by $\omega=x+i y$, where $x$ and $y$ are the Cartesians coordinates of $Q$ at plane 
$z=d$. In order to impose a uniform phase for the GO aperture field, all rays reflected from the main reflector must be parallel to the z-axis and, consequently, parallel to each other.

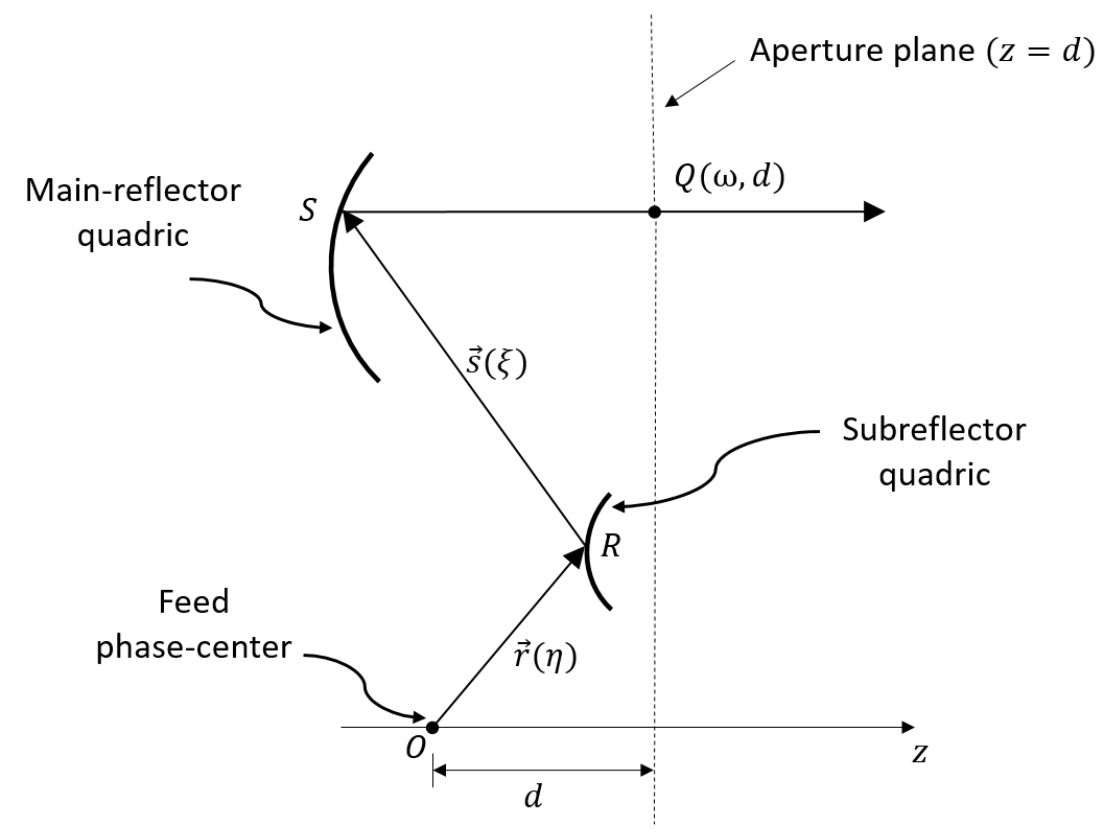

Fig. 3. Ray geometry.

\section{A. The mapping $\eta \rightarrow \omega$}

As demonstrated in [3], Snell's law at the subreflector point $R$ can be expressed as:

$$
L_{\eta}=\frac{1}{\xi-\eta}
$$

where $L_{\eta}$ is the first-order derivative with respect to $\eta$ of an auxiliary real function $L(\eta, \bar{\eta})$, which is obtained from the subreflector surface $r(\eta, \bar{\eta})$ and expressed as :

$$
e^{L}=\frac{r}{1+|\eta|^{2}}
$$

where $\bar{\eta}$ denotes the complex conjugate of $\eta$. When a uniform-phase field is required at the aperture plane, the mapping between source ray direction $\eta$ and aperture point $\omega$ is given by [2]:

$$
\omega=\eta A+\frac{A-2 e^{L}}{L_{\eta}}
$$

where $A=l-d$ is the total optical path length from origin $O$ (the source point) to the plane $z=0$. Consequently, a general point $R$ at the subreflector surface is located by the vector [4]:

$$
\vec{r}=e^{L}\left(2 \eta,|\eta|^{2}-1\right)
$$

Once the subreflector point $R$ is determined, the corresponding main-reflector point $S$ is straightforwardly located by [2]:

$$
\vec{r}+\vec{s}=\left\{\omega, \frac{|\omega|^{2}-A^{2}+2 e^{L}\left[A\left(1+|\eta|^{2}\right)-\bar{\eta} \omega-\eta \bar{\omega}\right]}{2\left(A-2 e^{L}\right)}\right\}
$$


To study the energy transfer from source to aperture, one must compare elementary areas subtended by ray tubes crossing a unit-radius sphere centered at $O$ (feed phase center location) and then, after two reflections, crossing the aperture plane, yielding an equation of energy conservation as in [4]:

$$
\frac{I(\eta)}{G(\omega)}=\left.\frac{\left(1+|\eta|^{2}\right)^{2}}{4}|| \omega_{\eta}\right|^{2}-\left|\omega_{\bar{\eta}}\right|^{2} \mid
$$

where $I(\eta)$ (watts/steradian) denotes the source radiated power density and $G(\omega)$ (watts/unit area) is the power density flowing normally to the aperture plane. Both $I(\eta)$ and $G(\omega)$ are given functions for the GO shaping process. For instance, the source pattern $I(\eta)$ can be tapered at the edge of the subreflector to reduce spillover losses, whereas the aperture power distribution $G(\omega)$ can be set uniform to maximize the aperture directivity, or tapered at the aperture edge to reduce side-lobe levels in the aperture's far-field radiation pattern.

Furthermore, in (9) $\omega_{\eta}$ and $\omega_{\bar{\eta}}$ are partial derivatives of $\omega$ with respect to $\eta$ and $\bar{\eta}$, respectively. Such derivatives are readily obtained from (6). Substituting $\omega_{\eta}$ and $\omega_{\bar{\eta}}$ into (9), one obtains the Monge-Ampère equation of elliptic (negative sign) or hyperbolic (positive sign) type in terms of $L(\eta)$ as [4]:

$$
\left|L_{\eta \eta}-L_{\eta}^{2}\right|^{2}-\left(L_{\eta \bar{\eta}}-\beta\right)^{2}= \pm B \frac{I(\eta)}{G(\omega)} \frac{4}{\left(1+|\eta|^{2}\right)^{2}}
$$

where

$$
\beta=\frac{-2 e^{L}\left|1+\eta L_{\eta}\right|^{2}}{l+d-2 e^{L}|\eta|^{2}} \quad B=\left(\frac{\left|1+\eta L_{\eta}\right|^{2}}{l+d-2 e^{L}|\eta|^{2}}\right)^{2}
$$

$L_{\eta \eta}$ is the second partial derivative of $L$ with respect to $\eta$ and $L_{\eta \bar{\eta}}$ is the second partial derivative of $L$ with respect to $\eta$ and its complex conjugate $\bar{\eta}$.

\section{B. Boundary conditions}

The Monge-Ampère equation (10) is to be solved as a boundary value problem. For that, we consider that the source at point $O$ radiates a conical tube of rays with a semi-vertex angle $\theta_{c}$ and axis tilted by $\theta_{0}$ from the z-axis, as illustrated in Fig. 4. After both reflections, the rays intersect the aperture plane within a given aperture contour (Fig. 4). The boundary condition is obtained by enforcing the cone edge-ray directions coming from the source to map, after two reflections, into points at the given aperture contour. In the present work, such contour is defined as a superellipse, described by [5]:

$$
\rho(\omega, \bar{\omega})=\left|\frac{\omega+\bar{\omega}-2 \omega_{c}}{2 r_{a_{x}}}\right|^{2 \sigma}+\left|\frac{\omega-\bar{\omega}}{i 2 r_{a_{y}}}\right|^{2 \sigma}
$$

where $\omega_{c}$ locates the aperture center lying at the $x$ axis. It is interesting to observe that for $\sigma=1$ the contour becomes an ellipse with widths $2 r_{a_{x}}$ and $2 r_{a_{y}}$, or a circle if $r_{a_{x}}=r_{a_{y}}$. For $\sigma \rightarrow \infty$ the contour becomes a rectangle with sides $2 r_{a_{x}}$ and $2 r_{a_{y}}$. 


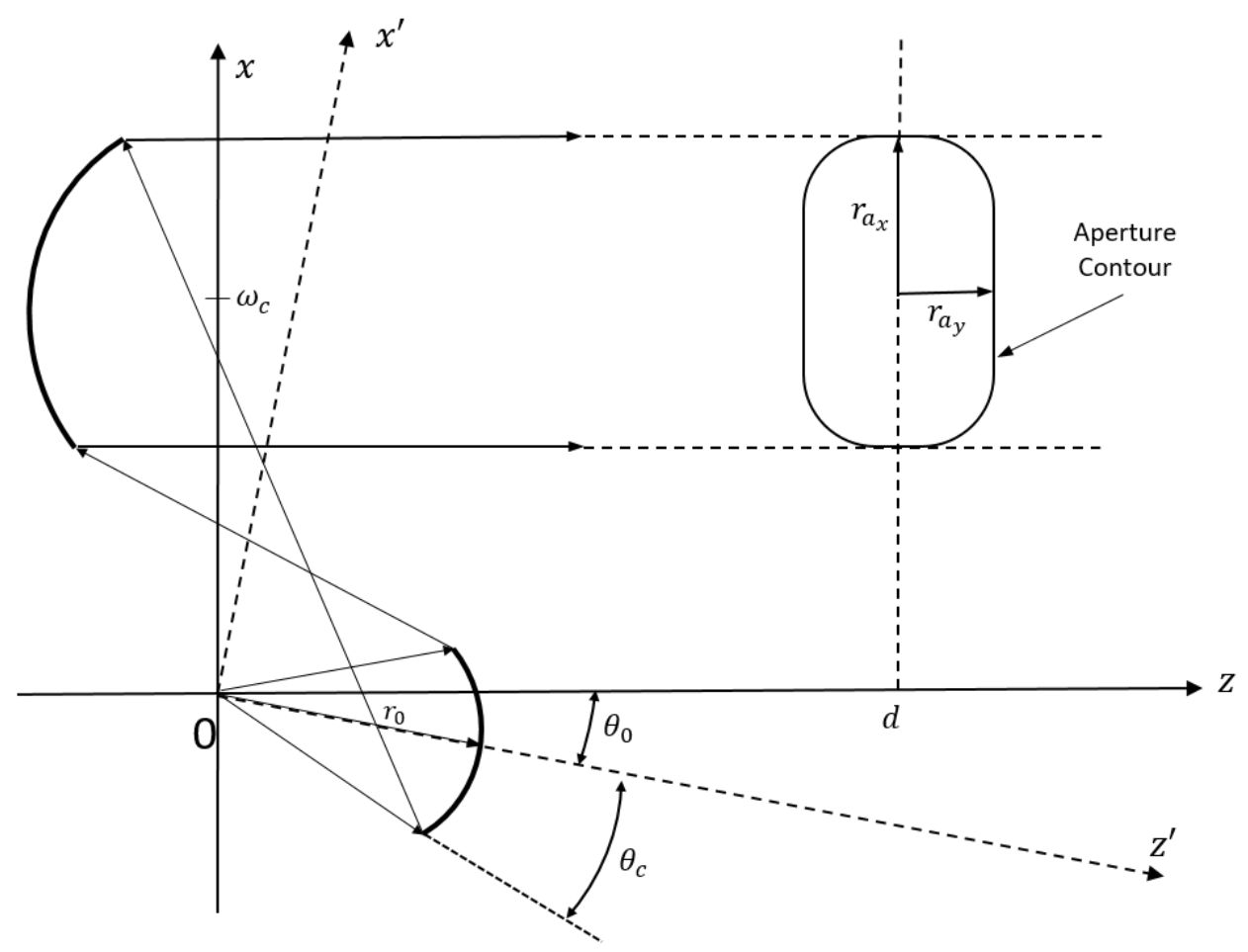

Fig. 4. Geometry of synthesis problem for an offset dual reflector with uniform phase at the aperture.

To impose the boundary condition in the present problem, we define a set of directions $\eta^{\prime}$ describing source rays at the periphery of the feed radiating cone. The relation between $\eta^{\prime}$ (i.e., direction with respect to the feed axis) and $\eta$ (same direction, but with respect to the principal coordinate system) is given by [2]:

$$
\eta^{\prime}=\frac{\eta_{0} \eta+1}{\eta_{0}-\eta} \rightleftarrows \eta=\frac{\eta_{0} \eta^{\prime}-1}{\eta_{0}+\eta^{\prime}}
$$

where $\eta_{0}=\cot \left(\theta_{0} / 2\right)$. Once the set of $\eta$ directions is determined, the corresponding aperture points $\omega$ are calculated from (6) and forced to satisfy (11).

\section{FORMULATION OF THE GO SHAPING PROCEDURE}

For the solution of the elliptical type of the Monge-Ampère equation (10), several iterative numerical methods have been employed, yielding a discrete representation of the reflector surfaces at a grid of points (or nodes). The first numerical technique applied in the solution of this problem [9] employed a 9-point cell finite-difference scheme to approximate the derivatives of $L(\eta)$ in (10). Alternatively, here we employ an axis-displaced confocal quadric (see Fig. 5) to locally represent the subreflector surface. Consequently, the surface derivatives in (10) can be analytically described in terms of the quadric' parameters, avoiding the use of approximate finite differences in the numerical solution. 


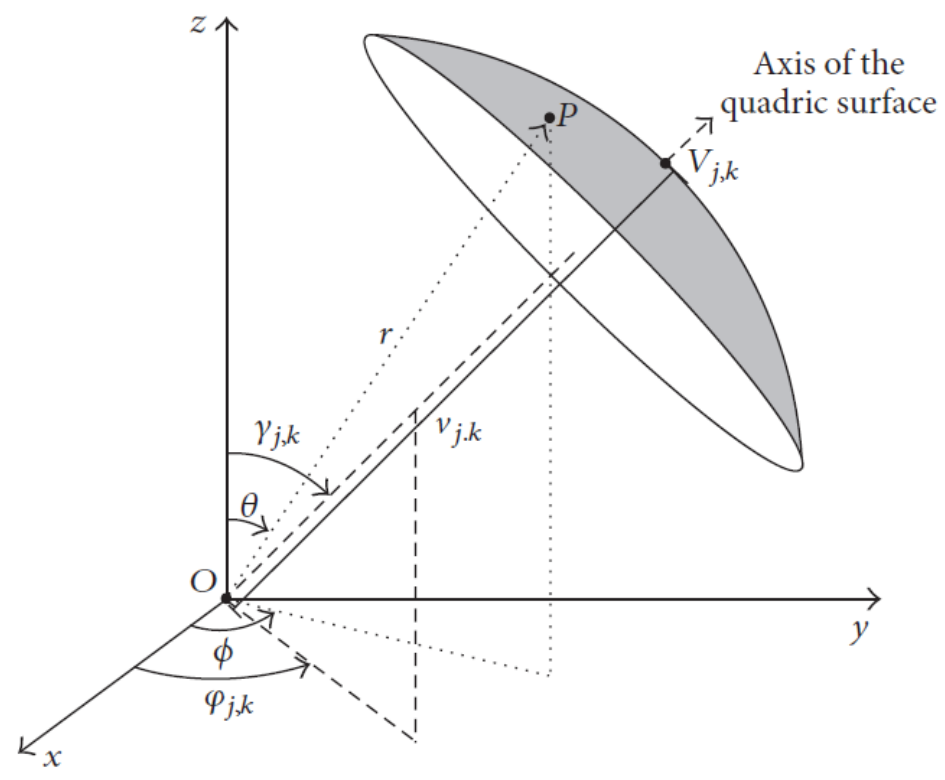

Fig. 5. Local axis-displaced confocal quadric [7].

With its vertex fixed at a given point $V$ at the subreflector surface, the expression of a confocal quadric surface (herein indexed by $j, k$ ) can be written in terms of the $\eta$ as in [7]:

$$
a_{j, k} e^{L(\eta)}=(\bar{\eta}+\eta) b_{j, k}+i(\bar{\eta}-\eta) c_{j, k}+\left(|\eta|^{2}-1\right) d_{j, k}-|\eta|^{2}-1
$$

where

$$
\begin{aligned}
a_{j, k} & =-e_{j, k} v_{j, k} \\
b_{j, k} & =e_{j, k} \sin \gamma_{j, k} \cos \varphi_{j, k} \\
c_{j, k} & =e_{j, k} \sin \gamma_{j, k} \sin \varphi_{j, k} \\
d_{j, k} & =e_{j, k} \cos \gamma_{j, k}
\end{aligned}
$$

being $a_{j, k}$ its semilatus rectum, $e_{j, k}$ its eccentricity, $v_{j, k}$ the distance between $O$ and the quadric vertex $V_{j, k}$, and $\varphi_{j, k}$ and $\gamma_{j, k}$ the azimuth and elevation angles of the quadric axis, respectively (see Fig. 5). Once the local representation of the subreflector is known, the derivatives of $L(\eta, \bar{\eta})$ in the vicinity of point $V_{j, k}$ can be analytically expressed. The first derivative of $L$ with respect to $\eta$ is given by [7]:

$$
L_{\eta}=-\frac{\left(d_{j, k}-1\right) \bar{\eta}_{j, k}+\left(b_{j, k}-i c_{j, k}\right)}{\left(d_{j, k}-1\right)\left|\eta_{j, k}\right|^{2}+\left(b_{j, k}-i c_{j, k}\right) \eta_{j, k}+\left(b_{j, k}-i c_{j, k}\right) \bar{\eta}_{j, k}-d_{j, k}-1}
$$

Substituting (13) and (15) into (6) ones obtains the mapping between the source-ray direction $\eta_{j, k}$ and the corresponding aperture point location $\omega_{j, k}$ :

$$
\omega_{j, k}=\frac{2 a_{j, k}+A\left[\left(d_{j, k}+1\right)-\left(b_{j, k}+i c_{j, k}\right) \bar{\eta}_{j, k}\right]}{\left(d_{j, k}-1\right) \bar{\eta}_{j, k}+\left(b_{j, k}-i c_{j, k}\right)}
$$

One of the main advantages of using confocal quadrics to locally represent the subreflector surface, besides avoiding the use of finite differences in the numerical solution, is that $\left|L_{\eta \eta}-L_{\eta}^{2}\right|=0$ for a quadric [2], yielding an important simplification of the Monge-Ampère equation (10): 


$$
-\left(L_{\eta \bar{\eta}}-\beta\right)^{2}= \pm B \frac{I\left(\eta_{j, k}\right)}{G\left(\omega_{j, k}\right)} \frac{4}{\left(1+\left|\eta_{j, k}\right|^{2}\right)^{2}}
$$

where $\beta$ and $B$ are rewritten as

$$
\begin{gathered}
\beta=\frac{2 e^{L}\left|L_{\eta}\right|^{2}}{2 e^{L}-A} \\
B=\frac{\left|L_{\eta}\right|^{4}}{\left(2 e^{L}-A\right)^{2}}
\end{gathered}
$$

$e^{L}$ and $L_{\eta}$ are written as in (13) and (15), respectively, while the second derivative $L_{\eta \bar{\eta}}$ is obtained by deriving (15) with respect to $\bar{\eta}[10]$ :

$$
L_{\eta \bar{\eta}}=\frac{b_{j, k}^{2}+c_{j, k}^{2}+d_{j, k}^{2}-1}{\left(d_{j, k}-1\right)\left|\eta_{j, k}\right|^{2}+\left(b_{j, k}-i c_{j, k}\right) \eta_{j, k}+\left(b_{j, k}-i c_{j, k}\right) \bar{\eta}_{j, k}-d_{j, k}-1}
$$

\section{NUMERICAL PROCEDURE}

Although the numerical scheme described above is not associated with any specific grid type, here a polar grid is employed to solve the Monge-Ampère equation (17). The grid is built in terms of the spherical angles $\theta^{\prime}$ and $\phi^{\prime}$ (with respect to the feed $z^{\prime}$-axis), which define the source-ray directions $\eta^{\prime}=\cot \left(\theta^{\prime} / 2\right) e^{i \phi^{\prime}}$. At each node $j, k$, the corresponding $\eta_{j, k}^{\prime}$ is associated with angles $\theta_{j}^{\prime}$ and $\phi_{k}^{\prime}$, being $\theta_{j}^{\prime}=\theta_{c}(j / J)^{\alpha}$ and $\phi^{\prime}{ }_{k}=2 \pi k / K$, where $j=1, \ldots, J$ and $k=1, \ldots, K$ index the grid rings and radials, respectively. The parameter $\alpha$ controls the variation of the rings' radii with respect to $\theta^{\prime}$. If $\alpha<1$, the distance between adjacent rings decreases with $j$, while for $\alpha>1$ such distance increases. This strategy was adopted to minimize distortions on central and peripheral rings which emerge whenever a superellipse aperture contour is adopted [7]. Afterwards, the source-ray direction $\eta_{j, k}$ with respect to the principal coordinate system is obtained from (12).

To solve for the reflector shaping, an operator $\Gamma\left[L_{j, k}\right]$ is defined at each inner grid node $j, k$ :

$$
\Gamma\left[L_{j, k}\right]=\left(L_{\eta \bar{\eta}}-\beta\right)^{2} \pm B \frac{I\left(\eta_{j, k}\right)}{G\left(\omega_{j, k}\right)} \frac{4}{\left(1+\left|\eta_{j, k}\right|^{2}\right)^{2}}
$$

where $e^{L}, L_{\eta}$ and $L_{\eta \bar{\eta}}$ are analytically defined as in (13), (15) and (19), respectively. The operator $\Gamma\left[L_{j, k}\right]$ simply enforces the solution of (17) at each inner node $j, k$ by obliging $\Gamma\left[L_{j, k}\right]=0$. For the nodes at the grid border (i.e., $j=J$ ), the boundary condition is enforced by defining the corresponding operator

$$
\Gamma\left[L_{J, k}\right]=\Gamma_{c}=\left|\frac{\omega_{J, k}+\bar{\omega}_{J, k}-2 \omega_{c}}{2 r_{a_{x}}}\right|^{2 \sigma}+\left|\frac{\omega_{J, k}-\bar{\omega}_{J, k}}{i 2 r_{a_{y}}}\right|^{2 \sigma}-1
$$

which obliges $\eta_{J, k}$ to satisfy (11) whenever $\Gamma\left[L_{J, k}\right]=0$. The application of (20) or (21) at each grid node leads to a system of $J \times K$ nonlinear equations in terms of $L\left[L_{1,1}, \ldots, L_{j, k}, \ldots, L_{J, K}\right]$. For the numerical solution of the system of nonlinear equations, Newton's method has been employed [2], 
[5], [6]. For the convergence and stability of the shaping technique, it is necessary an appropriate choice for the initial solution of the subreflector surface. In the present work, at all grid nodes, a single quadric is adopted as the initial solution, such that it produces an electric field with uniform phase at the aperture plane and its dimensions approximately specify the aperture contour. The algorithm converges when $\left|\Gamma\left[L_{j, k}\right]\right|<\varepsilon$ at all nodes, where $\varepsilon$ is a specified maximum value for the operator residues.

The local quadric surface at each node $j, k$ is uniquely determined once the four parameters $a_{j k}$, $b_{j k}, c_{j k}$, and $d_{j k}$ of (13) are calculated by imposing that the quadric matches the subreflector surface at the triangular 4-point cell around the grid node $j, k$, as depicted in Figure 6. This leads to analytical expressions in terms of $a_{j k}, b_{j k}, c_{j k}$, and $d_{j k}$ for the operators at the 4-point cell. The initial condition is imposed at the center of the grid $(j=1)$ by forcing the subreflector central point to be at a prescribed constant location, which controls the subreflector size. The particular cases of 4-point cells at the grid center and border are illustrated in Figs. 7(a) and (b), respectively.

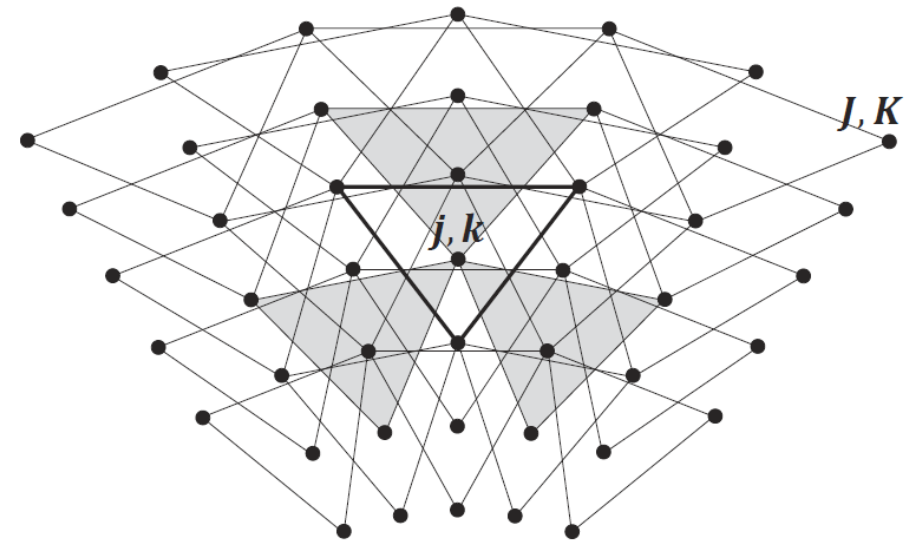

(a)

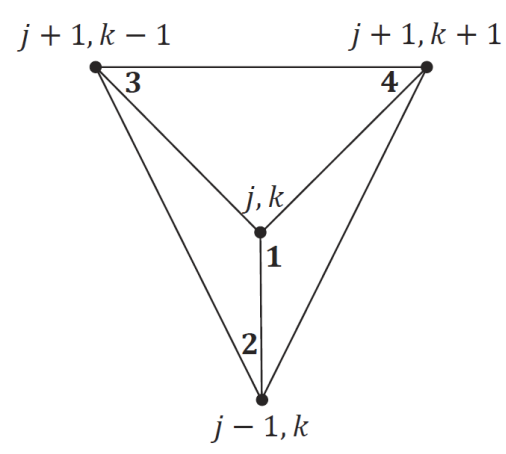

(b)

Fig. 6. (a) Polar grid with triangular cells. (b) Triangular cell with four points [10].

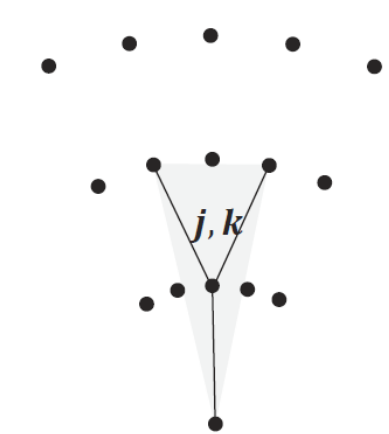

(a)

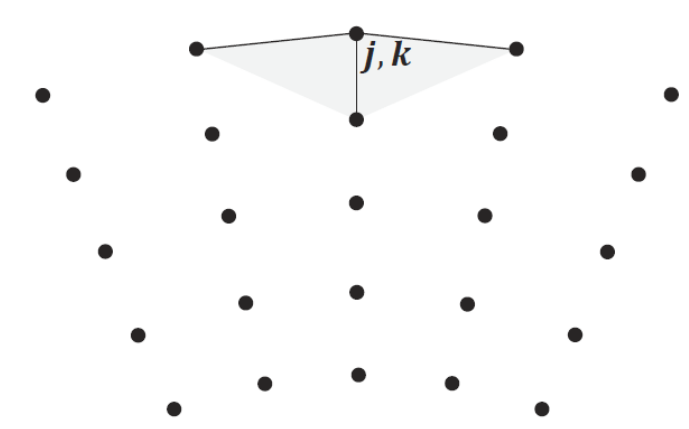

(b)

Fig. 7. (a) Triangular cell for the first grid ring. (b) Triangular cell for the last grid ring [10]. 


\section{CASE STUDY}

In this present case study, an offset dual reflector is designed to provide, within a superelliptical contour at the main-reflector aperture, a Gaussian power distribution $G(\omega)$ represented by

$$
G(\omega)=G_{0} e^{-\psi \rho(\omega, \bar{\omega})}
$$

where the parameter $\psi$ was specified to provide $6 \mathrm{~dB}$ attenuation at the aperture boundary and the superellipse $\rho(\omega, \bar{\omega})$ was defined as in (11). The superellipse was defined with $\sigma=1.6$ and widths $r_{a_{x}}=50 \mathrm{~cm}$ and $r_{a_{y}}=63.2 \mathrm{~cm}$. The aperture center was set over the $x$ axis with $\omega_{c}=70 \mathrm{~cm}$.

The feed radiation was modeled as a raised cosine $I(\eta)=I_{0}\left(\cos \theta^{\prime}\right)^{2 n}$ with $n=9$ to provide $12.88 \mathrm{~dB}$ attenuation at the subreflector edge. The constants $I_{0}$ and $G_{0}$ were specified such that the energy is conserved:

$$
\int_{0}^{2 \pi} \int_{0}^{\theta_{c}} I_{0}\left(\cos \theta^{\prime}\right)^{2 n} \operatorname{sen} \theta^{\prime} d \theta^{\prime} d \phi^{\prime}=\int_{0}^{2 \pi} \int_{0}^{\rho(\omega, \bar{\omega})} G_{0} e^{-\psi \rho(\omega, \bar{\omega})} \rho d \rho d \phi
$$

The feed cone has an offset angle $\theta_{0}=-12^{0}$ and half-angle $\theta_{c}=32^{0}$. Its field is vertically polarized. The distance from $O$ to the subreflector along the central ray of the polar grid was set to be $r_{0}=35 \mathrm{~cm}$ and the constant $A$ was fixed with $200 \mathrm{~cm}$. These specifications were chosen to avoid blockage and led to an offset antenna with Gregorian configuration, which results in a much more compact design [11], as illustrated in Fig. 9.

The initial solution $L^{(0)}$ for the numerical synthesis procedure was a quadric surface with $a_{0}=$ $-15.7641 \mathrm{~cm}, b_{0}=0.228153, c_{0}=0.0$ and $d_{0}=0.149907$. These initial solution's parameters were obtained imposing the feed cone angles $\theta_{0}=-12^{0}$ and $\theta_{c}=32^{0}$, together with $r_{a_{x}}=50$ $\mathrm{cm}$ and $\omega_{c}=70 \mathrm{~cm}$ in (16). Being $L^{(0)}$ an appropriate surface which closely approximates the final solution, the synthesis procedure introduced few curvature changes upon the initial solution, resulting in a dual reflector system with low cross-polarization levels [5].

For the GO synthesis, it was employed a nonuniform polar grid, with $\alpha=0.7, J=12$, and $K=$ 54 to obtain $\theta_{j}^{\prime}=\theta_{c}(j / J)^{\alpha}$ and $\phi^{\prime}{ }_{k}=2 \pi k / K$, as illustrated in Fig. 8(a). To guarantee convergence of the numerical shaping procedure, the aperture perimeter was gradually deformed from a circle to the specified superelliptical contour. The synthesis procedure converged after 15 iterations with $|\Gamma[\mathrm{L}]|_{\text {mean }}=8.88282^{-18}$ for inner points and $|\Gamma[\mathrm{L}]|_{\text {mean }}=2.70163^{-13}$ at the aperture perimeter, where $|\Gamma[\mathrm{L}]|_{\text {mean }}$ represents the mean difference between two successive solutions in the iterative process.

The results of the synthesis are shown in Figs. 8(b), which illustrates the intersection of the rays with the aperture plane $x y$, and Fig. 9, which shows the shaped sub- and main-reflectors together with ray tracing at the symmetry plane $y=0$. It is noted that the main-reflector dimensions in $\mathrm{x}$ and $\mathrm{y}$ directions are equal to aperture specifications, which were properly satisfied in the design procedure. This shows the effectiveness of the imposed boundary conditions. Furthermore, the concentration of rays close to the aperture's rim demonstrates the effectiveness of the reflector shaping in 
redistributing power density over the main reflector aperture. As the feed illuminates the subreflector edge with a $-12 \mathrm{~dB}$ attenuation, while a $-6 \mathrm{~dB}$ attenuation was demanded at the aperture's rim, the shaping procedure ended up concentrating rays close to the aperture's rim in order to compensate for the larger feed edge attenuation. This result is a direct consequence of the effectiveness of the MongeAmpère equation.

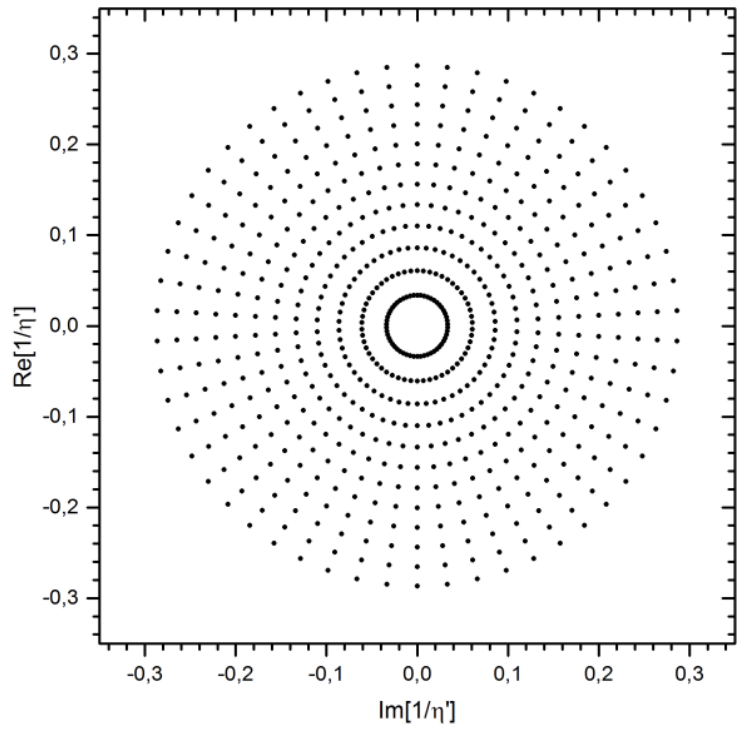

(a)

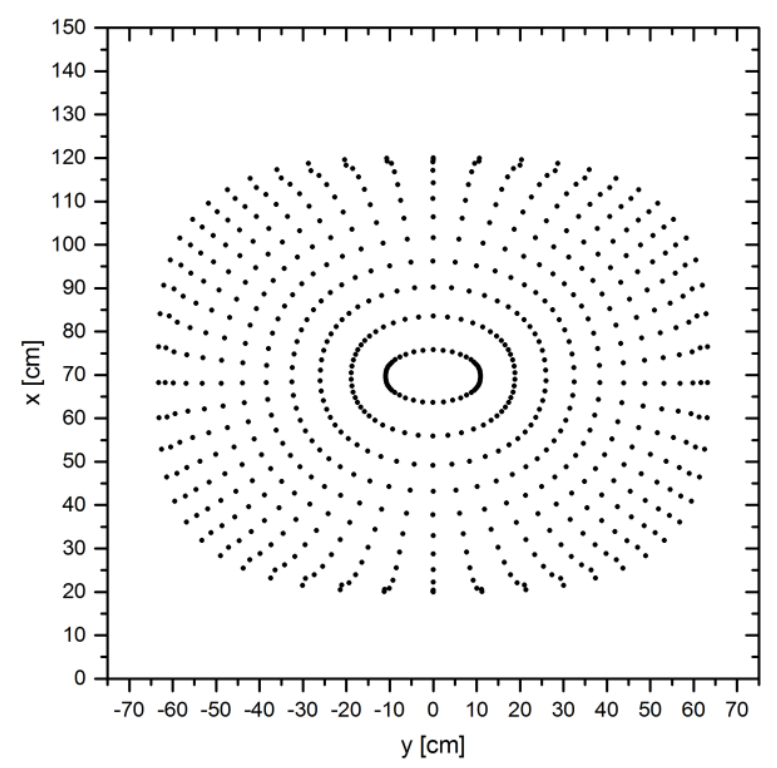

(b)

Fig. 8. Nonuniform polar grid representation $(\boldsymbol{\gamma}=\mathbf{0 . 7})$ of the ray directions at the (a) feed complex plane $\boldsymbol{\eta}^{\prime}$ and (b) aperture plane $\boldsymbol{\omega}=\boldsymbol{x}+\boldsymbol{i} \boldsymbol{y}$.

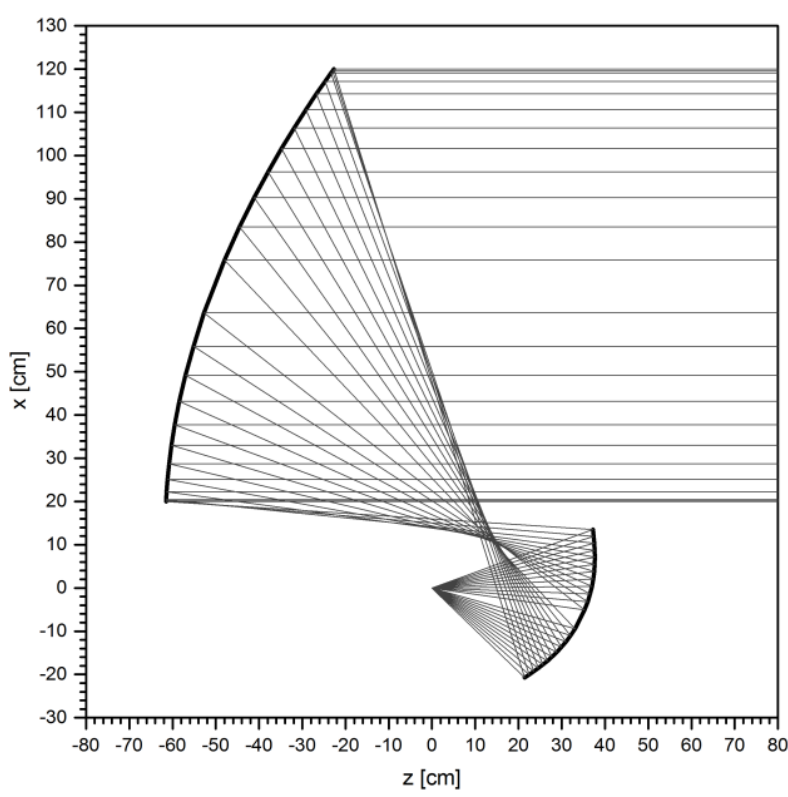

Fig. 9. Shaped offset dual-reflector surfaces and ray tracing at the symmetry plane.

In order to verify the achievement and usefulness of the proposed GO shaping technique, the surface points obtained by the synthesis were interpolated by quintic pseudo-splines [12], to obtain a smooth numerical representation of the reflectors' surfaces, and the resulting shaped dual-reflector 

DOI: http://dx.doi.org/10.1590/2179-10742020v19i2813

antenna was analyzed by the Physical Optics (PO) method with equivalent edge currents [13]. The analysis was conducted at $11.725 \mathrm{GHz}$, which provides dimensions with, at least, 13.38 wavelengths for the subreflector surface, large enough to validate the GO principles adopted in the shaping procedure. The PO radiation patterns are shown in Figs. 10 and 11. Figure 10 presents the antenna radiation patterns at the principal planes $\phi=0^{0}$ (the symmetry E-plane) and $\phi=90^{\circ}$ (H-plane), whereas Figs. 11(a) and 11(b) illustrate the contour plots of the PO radiation patterns in the $u v$-plane for the principal polarization and cross-polarization, respectively.

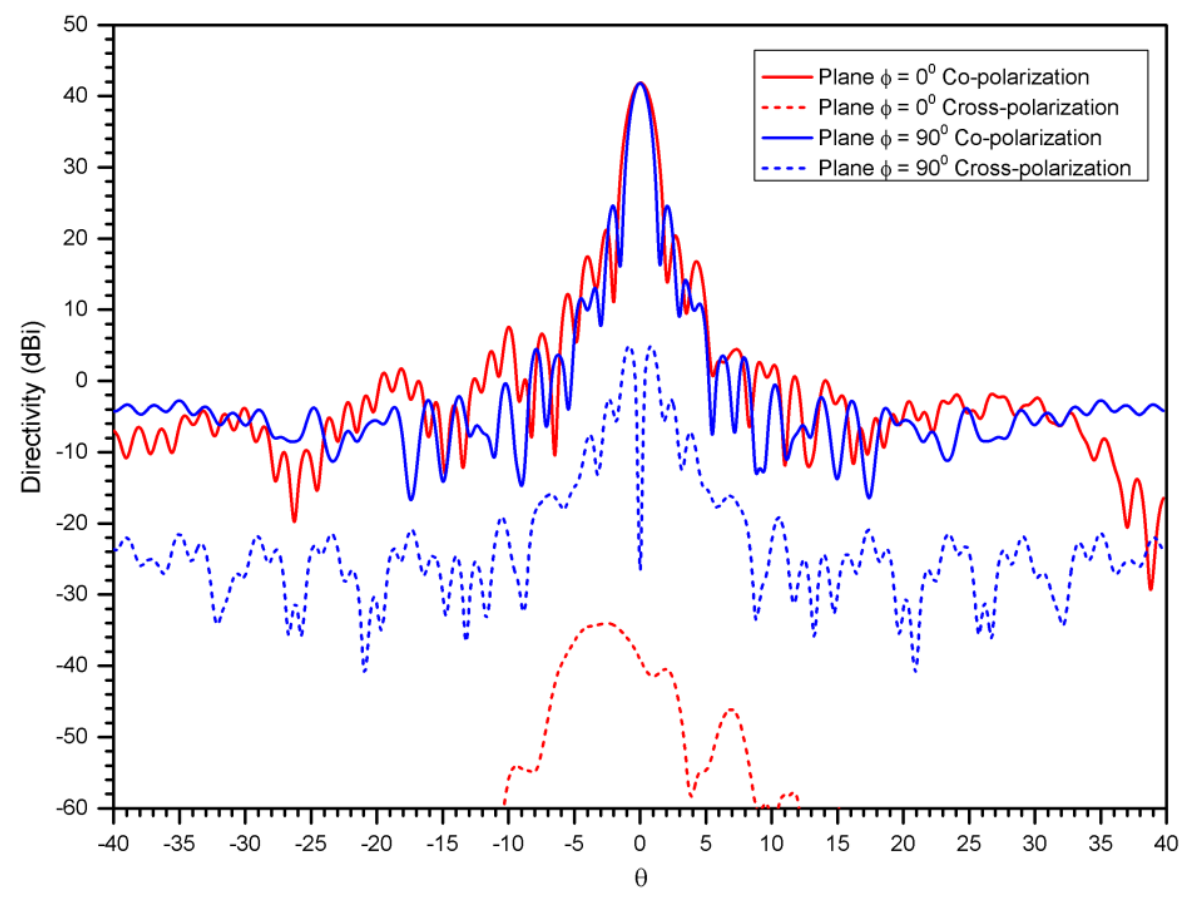

Fig. 10. Radiation patterns of the shaped offset dual reflectors at $11.725 \mathrm{GHz}$.

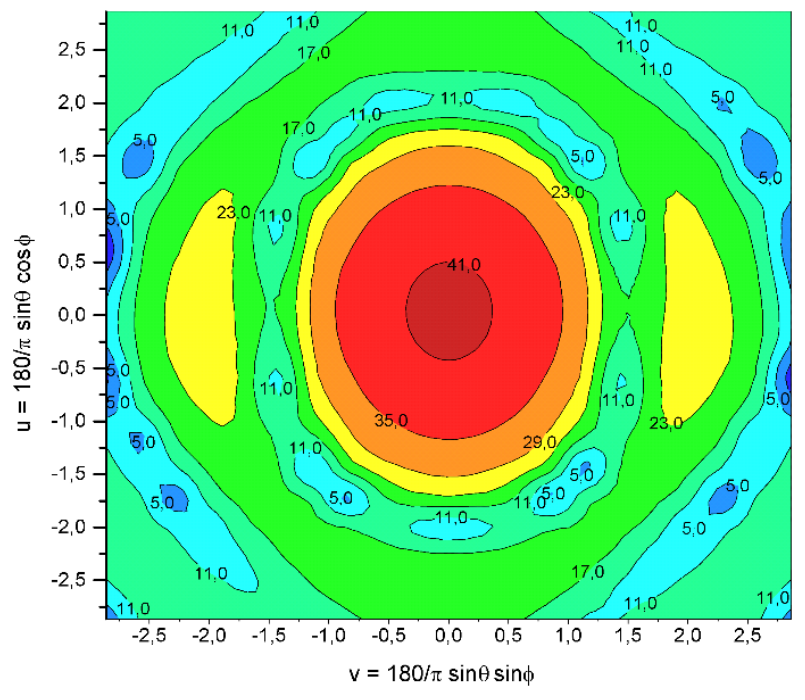

(a)

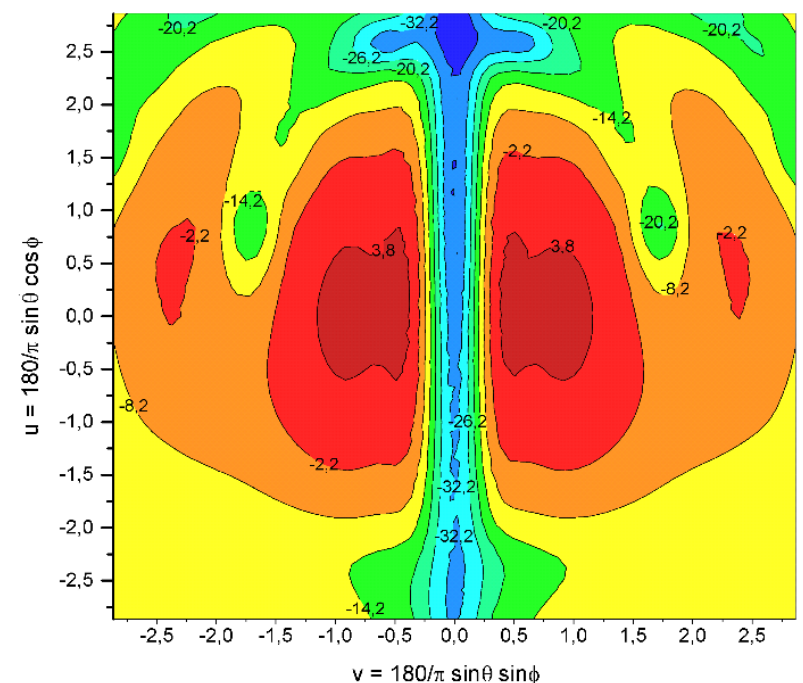

(b)

Fig. 11. (a) Co-polar and (b) cross-polar radiation patterns in the $u v$-plane at $11.725 \mathrm{GHz}$. 
From the results depicted in Fig. 10, it can be noted that the maximum directivity occurs at $\theta=$ $0^{0}$, as expected, with a value of $41.93 \mathrm{dBi}$. The first sidelobe level has an amplitude of $17.04 \mathrm{dBi}$ on plane $\phi=0^{0}$ and $25.03 \mathrm{dBi}$ on plane $\phi=90^{\circ}$, indicating an attenuation of $-24.89 \mathrm{~dB}$ and -16.90 $\mathrm{dB}$, respectively, with respect to the main beam. The maximum cross-polarization levels are -34.00 $\mathrm{dBi}$ and $5.10 \mathrm{dBi}$ on planes $\mathrm{E}$ and $\mathrm{H}$, respectively. The algorithm was able to design a dual-reflector antenna with high gain, low sidelobe levels, and low cross-polarization levels. These results corroborate the choices for a tapered source pattern $I(\eta)$ at the edge of the subreflector to reduce spillover losses, an aperture power distribution $G(\omega)$ tapered at the aperture edge to reduce side lobelevels and an initial solution $L^{(0)}$ close to the final one to reduce cross-polarization levels. And, as expected from Fig. 8(a), the far-field pattern has approximately an elliptical main-beam on the $u v$ plane, which is narrower in the plane corresponding to the largest aperture dimension.

\section{CONCLUSIONS}

The GO synthesis of dual-reflector antennas has been detailed examined by many authors [2], [5]. However, the present work investigated an alternative numerical scheme where the subreflector surface is locally described by axis-displaced confocal quadrics, allowing the analytical representation of the derivatives appearing in the Monge-Ampère equation and its subsequent simplification. Such analytical representation provides superior accuracy for the calculation of derivatives, which is not possible in previous techniques employing finite differences. This alternative approach was recently proposed in [7] to synthesize single offset reflectors and here the technique was extended to the synthesis of offset dual-reflector antennas.

To illustrate the effectiveness of the present methodology, a case study was designed to provide Gaussian aperture power distribution with a superelliptical contour in a dual-reflector Gregorian configuration. Although not discussed in this work, the algorithm is also capable of handling Cassegrain configurations as well. The subsequent PO analysis presented an excellent agreement with the required GO specifications. The method proved to be fast and stable, achieving convergence with relatively few iterations. Due to the excellent performance of the present procedure in reducing discretization errors, the methodology may become a promising technique to establish numerical solutions of surface synthesis problems modeled by nonlinear Monge-Ampère equations, like those appearing in lens's designs.

\section{ACKNOWLEDGMENT}

This work was partially supported by CNPQ and CAPES (PROCAD 068419/2014-01).

\section{REFERENCES}

[1] S. L. Avila, W. P. Carpes Jr., J. R. Bergmann, "Satellite reconfigurable contour beam reflector antennas by multiobjective evolutionary optimization", Journal of Microwaves, Optoelectronics and Electromagnetic Applications, vol. 7, no. 2, pp. 101-114, 2008.

[2] B. S. Westcott, F. A. Stevens, and F. Brickell, "GO synthesis of offset dual reflectors", IEE Proceedings H: Microwaves, Optics and Antennas, vol. 128, no. 1, pp. 11-18, 1981. 
[3] F. Brickell, L. Marder, and B. S. Westcott, "The geometrical optics design of reflectors using complex coordinates", Journal of Physics A: General Physics, vol. 10, pp. 245-260, 1977.

[4] F. Brickell, L. Marder, and B. S. Westcott, "Phase and power density distributions on plane apertures of reflector antennas", Journal of Physics. A. Mathematical and General, vol. 11, no. 4, pp. 777-789, 1978.

[5] J. R. Bergmann, The geometrical optics design of dual reflector antennas, Ph.D. Thesis, Queen Mary University of London, London, UK, 1986.

[6] J. Bergmann, R. C. Brown, P. J. B. Clarricoats, H. Zhou, "Synthesis of shaped-beam reflector antenna patterns", IEE Proceedings H: Microwaves, Optics and Antennas, vol. 135, no. 1, pp. 48-53, 1988.

[7] R. A. Penchel, J. R. Bergmann, and F. J. S. Moreira, "Shaping single offset reflector antennas using local axis-displaced confocal quadrics", International Journal of Antennas and Propagation, vol. 2016, pp. 1-9, 2016.

[8] B. S. Westcott, Shaped reflector antenna design, Research Studies Press Ltd., Hertfordshire, U.K., 1983.

[9] A. P. Noris, B. S. Westcott, "Computation of reflectors surfaces for bivariate beam-shaping in the elliptic case", Journal of Physics A: Mathematical and General, vol. 9, no. 12, pp. 2159-2169,1976.

[10] R. A. Penchel, Síntese de antenas refletoras utilizando seções cônicas e superfícies quádricas confocais, $\mathrm{PhD}$ Thesis, PUC-Rio, Rio de Janeiro, BR, 2014.

[11] M. Terada, "Dual offset multibeam antennas for satellite applications", Journal of Microwaves and Optoelectronics, vol. 3, no. 2, pp. 40-45, 2003.

[12] J. R. Bergmann, F. J. V. Hasselmann, F. L. Teixeira, and C. G. Rego, "Comparison between techniques for global surface interpolation in shaped reflector analysis”, IEEE Transactions on Antennas and Propagation, vol. 42, no. 1, pp.47-53, 1994.

[13] A. Michaeli, "Equivalent edge currents for arbitrary aspects of observation", IEEE Transactions on Antennas and Propagation, vol. 32, pp. 252-258, 1984. 